\title{
Modeling LRRK2 Parkinsonism
}

\author{
Kelly Hinkle and Heather Melrose \\ Dept. Of Neuroscience, Mayo Clinic Jacksonville, \\ USA
}

\section{Introduction}

Mutations in the LRRK2 gene were first described in 2004 and have now emerged as the most important genetic finding in Parkinson's Disease (PD) (Paisan-Ruiz et al., 2004, Zimprich et al., 2004). Incredibly, they account for up to $40 \%$ of familial parkinsonism in certain ethnic populations (Ishihara et al., 2007; Kachergus et al., 2005; Ozelius et al., 2006). Moreover LRRK2 mutations also are responsible for about $2 \%$ of PD in sporadic (idiopathic) patients and two risk factors increase the risk for sporadic PD in Asian populations by twofold (Di Fonzo et al., 2006; Farrer et al., 2007; Ross et al., 2008; Tan, 2006). While the precise functional role of LRRK2 protein is still emerging, it has attracted intense pharmaceutical interest as a highly "druggable" target. This, and other unique properties including variable penetrance and pathologies that overlap with other neurodegenerative disorders, have fueled theories that a LRRK2 therapeutic will benefit not just familial parkinsonism, but also sporadic parkinsonism and perhaps even neurodegeneration in general. Development of LRRK2 agents relies on the availability of animal models, which provide not only information of physiological and pathological functions but also a means for testing and phenotypic reversal. In this chapter we summarize the progress to date for both invertebrate and vertebrate models of LRRK2 parkinsonism and briefly discuss opportunities for therapeutic development.

\section{Background}

\subsection{LRRK2 mutations}

There are five LRRK2 mutations (R1441C, R1441G; Y1699C; G2019S; I2020T) proven to segregate with disease and cause pathogenicity (Paisan-Ruiz et al., 2004; Zimprich et al., 2004) and two variants that are considered risk factors (LRRK2 G2385R and R1628P) (Farrer et al., 2007; Ross et al., 2008; Tan, 2006). While all leading to the same disease, the substitutions are not all found in the same functional domain. LRRK2 R1441C and G substitutions affect the Roc domain, a 'Ras-like' part of the protein (Mata et al., 2005). The LRRK2 Y1699C substitution and the R1628P risk factor are found in the COR domain and LRRK2 G2019S and I2020T are encoded within exon 41 at the 'activation hinge' of the MAPKKK domain (Mata et al., 2005). To date, the only substitution affecting risk of disease in the WD40 domain is the G2385R risk factor. The most common substitution, LRRK2 G2019S, originates from a common founder and presents with variable, age-dependent penetrance (Healy et al., 2008; Hulihan et al., 2008; Kachergus et al., 2005). 


\subsection{Clinical and neuropathological features of LRRK2 parkinsonism}

The overall clinical profile in individuals with LRRK2 mutations is similar to that of typical late-onset PD (Aasly et al., 2005; Djaldetti et al., 2008; Healy et al., 2008). In agreement with observations in idiopathic PD, individuals with LRRK2-parkinsonism are generally responsive to levodopa treatment. While tremor, bradykinesia, and rigidity occurs in individuals with idiopathic PD, tremor is more frequently observed in individuals with LRRK2 G2019S (Djaldetti et al., 2008; Healy et al., 2008; Hulihan et al., 2008).

Disease duration, severity and response to treatment do not differ between G2019S carriers, regardless of zygosity (Ishihara et al., 2007, (Djaldetti et al., 2008) Individuals harboring LRRK2 G2019S present with similar non-motor features to idiopathic PD with the exception of a lower rate of cognitive impairment and hyposmia (Healy et al., 2008; Hulihan et al., 2008). Remarkably, there are aged G2019S carriers that escape disease (Carmine Belin et al., 2006; Kay et al., 2005). The LRRK2 G2019S mutation is primarily associated with transitional or brainstem Lewy body pathology, reminiscent of typical, late-onset idiopathic PD (Ross et al., 2006). LRRK2 mutation carriers presenting with tauopathy or ubiquitin-positive inclusions, with nigral neuronal loss and gliosis, have also been described (Wszolek et al., 2004; Dachsel et al., 2007; Rajput et al., 2006) Neuropathologic studies of affected individuals from other PARK8-linked kindreds, including Family A (Y1699C), Family D (R1441C), and the Sagamihara family (I2020T) have presented with pleomorphic pathology, including neuronal loss without co-existing pathology or with a-synuclein, ubiquitin-positive inclusions or tau pathology (Taylor et al., 2006).

\subsubsection{LRRK2 expression}

LRRK2 mRNA is highly expressed in the lungs, kidney, spleen and leucocytes; (Biskup et al., 2006; Li et al., 2007; Westerlund et al., 2008; White et al., 2007; Zimprich et al., 2004) however, its expression profile suggests that LRRK2 is unlikely to be an essential developmental protein (Biskup et al., 2007). In adult rodent brain, LRRK2 mRNA is somewhat restricted, with highest levels found in dopamine receptive areas but surprisingly low levels in the dopamine synthesizing areas (Biskup et al., 2006; Galter et al., 2006; Melrose et al., 2006; Simon-Sanchez et al., 2006; Taymans et al., 2006). LRRK2 protein levels do not entirely correlate with mRNA levels, suggesting transport following synthesis. LRRK2 protein is highly expressed in the spiny neurons in the striatum and in the dopamine neurons of the substantia nigra, (Biskup et al., 2006; Melrose et al., 2007) but robust expression is also found in many non-dopaminergic areas throughout the brain, for example the hippocampus and cerebellum (Biskup et al., 2006; Greggio et al., 2006; Higashi et al., 2007a; Higashi et al., 2007b; Melrose et al., 2007; Miklossy et al., 2006).

\subsection{Predicted function}

LRRK2 protein contains a GTPase domain as well as a kinase domain containing homologous sequence to tyrosine-like kinase (TLK), including mixed-lineage kinases (MLKs) and receptorinteracting kinases (RIPKs) (Mata et al., 2006). Given the size of the protein monomer (286kDa), its protein-protein interaction domains (ANK, LRR, WD40) and its propensity to dimerize, (Greggio et al., 2008) it is reasonable to postulate LRRK2 is part of a larger scaffolding complex. While in silico modeling suggests that LRRK2 mutations may have increased kinase activity and 
act through a dominant "gain-of-function", data on kinase activity across the different mutations has been controversial, with only the G2019S mutation consistently reported to enhance kinase activity compared to wild-type activity (Adams et al., 2005; Jaleel et al., 2007; Luzon-Toro et al., 2007; MacLeod et al., 2006; Smith et al., 2006; West et al., 2005).

LRRK2 has been characterized extensively in vitro. Although it is beyond the scope of this review to discuss these studies detail, several lines of evidence several lines of evidence point to a role in synaptic function (possibly by regulation of vesicle synthesis or transport and/or regulation of membranous structure) and as a regulator of neuronal outgrowth and guidance.

\section{Invertebrate models}

\subsection{LRRK2 worm models}

LRK-1, the paralogue of LRRK2 in Caenorhabditis elegans, shares $~ 35 \%$ identity and $57 \%$ similarity with the enzymatic GTPase and kinase domains (Samann et al., 2009) and is expressed ubiquitously, including in muscle, intestine and importantly in neurons (Sakaguchi-Nakashima et al., 2007; Samann et al., 2009). Experimental approaches to study LRRK2 in worms have included loss of LRK-1 function, over-expression of LRK-1 and transgenic insertion of human wild type and mutant LRRK2.

\subsubsection{LRK-1 loss of function worm models}

Sakaguchi-Nakashima et al first described loss-of-function LRK-1 mutants, the km17 line, which has a deletion in the kinase and WD40 domains, and km41 line that has the GTPase and kinase domain deleted (Sakaguchi-Nakashima et al., 2007). A third deletion strain, tm1898 (also lacking the GTPAse and kinase domain) was later described (Saha et al., 2009). All three deletion mutants result in mislocalization of synaptobrevin to dendrites, suggesting that normally LRK-1 plays a role in sorting and/or localization of synaptic vesicle proteins (Sakaguchi-Nakashima et al., 2007; Samann et al., 2009) possibly by excluding them from dendrite-specific transport machinery in the Golgi (SakaguchiNakashima et al., 2007). Loss-of-function LRK-1 ( $\mathrm{km} 17$ and $\mathrm{km} 41)$ worms also exhibited defects in movement and chemotaxis (Sakaguchi-Nakashima et al., 2007). Both the km41 and tm1898 mutants were reported to have increased sensitivity to endoplasmic reticiulum stress, following tunicamycin exposure but were resistant to mitochondrial stress via paraquat (Samann et al., 2009). Conversely, Saha reported that RNAi knockdown of LRK-1, while not affecting baseline viability, did sensitize nemotodes to mictochrondrial dysfunction via rotenone treatment, a response that was confirmed in the km17 deletion line (Saha et al., 2009). Interestingly, loss of LRK-1 has been found to suppress the neurite outgrowth defects, mitochondrial abnormalities and increased paraquat sensitivity in PINK1 loss-of-function mutants. Additionally, the increased sensitivity of LRK-1 loss-of-function worms to tunicamycin was reduced in PINK-1 loss-of-function mutants, suggesting an antagonistic role of PINK-1 and LRK-1 (Samann et al., 2009). Curiously, loss of LRK-1 also attenuates transgenic LRRK2-induced DA neurodegeneration and basal slowing behavioral phenotype (Yao et al., 2010)

\subsubsection{Transgenic worm models}

Transgenic worms have been created by either overexpressing wild-type (WT) LRK-1 and/or comparable mutant LRK-1 or by overexpressing human WT or mutant LRRK2 with 
fluorescent tags (Sakaguchi-Nakashima et al., 2007; Samann et al., 2009) driven by tissuespecific promoters whereas others have over-expressed human LRRK2 WT and pathogenic mutants via the dopamine neuron-specific DAT promoters (Saha et al., 2009; Wolozin et al., 2008; Yao et al., 2010).

Over-expression of endogenous WT and mutant LRK-1 in neurons resulted in axon guidance defects and embryonic lethality (Samann et al., 2009). Surprisingly, worms expressing human LRRK2 had increased longevity and reduced vulnerability to mitochondrial toxicity, with human WT LRRK2 offering more protection than mutant G2019S, R1441C or R1441C kinase dead LRRK2 (Saha et al., 2009; Wolozin et al., 2008). Despite this protection, transgenic LRRK2 WT and G2019S worms exhibited DA neuron degeneration and decreased dopamine levels, with G2019S mutants exhibiting more severe phenotypes (Saha et al., 2009). These findings were confirmed by Yao et al, who also demonstrated that G2019S and R1441C mutant worms displayed progressive behavioral and locomotor dysfunction, which could be rescued by exogenous dopamine (Yao et al., 2010).

Taken together, the data from worm models of LRRK2 suggests that WT LRK/LRRK2 may work in a gain-of-function manner which is antagonistic to DA neuronal survival. While it does seem likely that LRK-1 is linked to mitochondrial pathways in some manner, the contrasting data makes any inferences difficult.

\subsection{Fruit fly models}

The Drosophila melanogster paralogue of human LRRK2, dLRRK (CG5483) has similar GTPase and kinase domains among others, with $46 \%$ and $44 \%$ homology respectively. Expression of LRRK is ubiquitous in the adult fly brain, which suggests that Drosophila is a good model for LRRK2 (Imai et al., 2008). As with the worm models, fly modelers have employed a number of similar experimental approaches including deletion and manipulation of dLRRK and overexpression of WT and mutant human LRRK2.

\subsection{1 dLRRK loss of function}

Several groups have used the loss of function e03680 line which has a piggyback transposon that results in kinase-null dLRRK2 (Imai et al., 2008; Kanao et al., 2010; Lee et al., 2010b; Lee et al., 2007; Lin et al., 2010; Tain et al., 2009; Wang et al., 2008) or a chromosomally deficient line dLRRKdf (Imai et al., 2008; Lee et al., 2007). Additionally, loss of dLRRK activity has been achieved by RNAi knock-down (Gehrke et al., 2010; Imai et al., 2008) and by 3KD kinase-dead mutation (Imai et al., 2008; Kanao et al., 2010; Lee et al., 2010b). Results have been contrasting, even between groups using the same line. For example, the e03680 line was reported to have no loss of dopaminergic neurons (Wang et al., 2008, Imai et al , 2008), however locomotive impairment (Lee et al., 2007; Tain et al., 2009), degenerative changes in DA neurons (Lee et al., 2007), irregular nerve terminal morphology/overgrowth and changes in neurotransmission (Lee et al., 2010b) were also reported in this line. Interestingly, Imai and colleagues did report increased levels of dopamine in the e03680 line, supporting neurotransmission or storage changes (Imai et al., 2008). Additionally, heterozygous e03680 flies expressing toxic Tau-GFP had longer dendrites and branching and decreased Tau inclusions (Lin et al., 2010).

Data on second hit response has also been contradictory, with the e03680 flies exhibiting increased (Wang et al., 2008) and decreased sensitivity (Imai et al., 2008) although both groups reported resistance of this line to paraquat. Chromosomally deficient line dLRRKdf 
was also resistant to hydrogen peroxide, and RNAi knockdown of LRRK2 decreased sensitivity to both hydrogen peroxide and paraquat (Imai et al., 2008).

\subsubsection{Transgenic fly models}

Many transgenic models of Drosophila employ the bipartite GAL4/UAS system (Brand and Perrimon, 1993) giving rise to tissue-specific gene expression. Some groups have used dLRRK WT and comparable mutant ectopic expressors utilizing GAL4 drivers directed to pan neuronal- and dopaminergic neuronal-specific expression among others (Gehrke et al., 2010; Imai et al., 2008; Kanao et al., 2010; Lee et al., 2010b; Lee et al., 2007; Lin et al., 2010). Some studies showed no deleterious effects, such as with locomotion or DA neuronal changes (Lee et al., 2007), whereas others showed that dLRRK mutants and/or WT overexpressors had decrease in dopamine and DA neurons (Imai et al., 2008) decreased dendrite arborization (Lee et al., 2010b; Lin et al., 2010) and neuromuscular junction (NMJ) bouton loss (Lee et al., 2010b). Other groups have utilized transgenic human LRRK2 GAL4driven Drosophila models (Gehrke et al., 2010; Kanao et al., 2010; Lee et al., 2010b; Lin et al., 2010; Liu et al., 2008; Ng et al., 2009; Venderova et al., 2009). Significant retinal degeneration and other eye abnormalities were observed in eye-specific hLRRK2 WT- and mutantexpressing cells (Liu et al., 2008; Venderova et al., 2009) whereas others did not observe this effect (Kanao et al., 2010; Ng et al., 2009).

Flies with ectopic neuronal expression of mutant and/or WT transgenes exhibited locomotive impairment and DA neuron loss (Lin et al., 2010; Liu et al., 2008; Ng et al., 2009; Venderova et al., 2009) and decreased boutons, NMJ length and arborization (Lee et al., 2010b). Endogenous dLRRK (Imai et al., 2008) and transgenic WT hLRRK2 (Ng et al., 2009) protein appeared punctate and localized to the cytoplasm and exogenous dLRRK colocalized to endosomal structures that partially overlapped with synaptic markers (Imai et al., 2008). Interestingly, transgenic mutant LRRK2 tended to form intracellular aggregates (Ng et al., 2009). Lin et al expressed hLRRK2 WT and mutants in Drosophila with GAL4 drivers specific for DA neuron dendrites and showed their degeneration (Lin et al., 2010). The hLRRK2 G2019S fly showed the most severe defect, including aberrant localization of axonal proteins, including microtubule-associated protein tau, to dendrites. Dendrite degeneration was exacerbated by overexpression of tau and conversely rescued by knockdown of tau in the transgenic mutant flies, this toxic effect being attributed to increased tau phosphorylation phosphorylation through Sgg (GSK3b homologue) (Lin et al., 2010). Both dLRRK mutants and/or WT overexpressors were significantly sensitive to oxidative stressors paraquat and hydrogen peroxide (Imai et al., 2008). Additionally, transgenic hLRRK WT (Venderova et al., 2009) and mutant (Ng et al., 2009) flies had increased sensitivity to mitochondria Complex I toxin rotenone and WT hLRRK2 flies had a decrease in the number of mitochondria at presynaptic nerve terminals (Lee et al., 2010b).

Taken together, dLRRK2 appears to have some role in the maintenance of dopaminergic neurons and the over-expression of LRRK2 results in locomotive impairment, DA neuron loss, decreased dendrite arborization, protein sorting or trafficking defects and increased sensitivity to oxidative stress. Although mortality rates differed among the studies, most groups saw that the hLRRK2 pathogenic mutants tended to have a more severe phenotype, providing evidence for the gain-of-function hypothesis. Like the worm models, the second hit data, while somewhat contrasting, do go some way to support LRRK2 involvement in stress response and mitochondrial pathways. 


\subsubsection{Fly interactor studies}

Studies have been performed utilizing double transgenic hLRRK2 flies concomitantly expressing human PINK1, DJ-1 or Parkin. These fly models exhibited various eye abnormalities and some had decreased DA neuron survival (Venderova et al., 2009) whereas coexpression of Parkin with mutant LRRK2 provided significant protection against DA neuron degeneration that occurs with age or in response to rotenone ( $\mathrm{Ng}$ et al., 2009) Also, loss-of-function dLRRK suppressed Parkin and PINK1 pathology in flies (Tain et al., 2009). Thus, this data further supports the observations that wild-type $d$ LRRK negatively regulates DA neuron survival and provides evidence that there is some sort of relationship between LRRK2 and these other PD-associated genes.

Drosophila models have been utilized for the search and characterization of LRRK2 substrates and/or interacting proteins (Imai et al., 2008; Lee et al., 2010b; Tain et al., 2009; Venderova et al., 2009). Imai and colleagues showed that both Drosophila and human LRRK2 phosphorylate 4E-BP (a negative regulator of translation and mediator of stress response), and that pathogenic mutations caused hyper-phosphorylation of $4 \mathrm{E}-\mathrm{BP}$, which led to reduced resistance to oxidative stress and increased dopaminergic neurodegeneration in flies (Imai et al., 2008). Likewise, loss-of-function e03680 flies had a decrease in the amount of phosphorylated 4E-BP (Tain et al., 2009). This association was confirmed in a study that not only showed that LRRK2 interacted with 4E-BP at the postsynaptic compartment, but also found that hLRRK2 phosphorylated Futsch (MAP1b homolog) and possibly interacted with Tubulin (Lee et al., 2010b). Another study found that dLRRK/hLRRK2 phosphorylated FoxO (a transcription factor involved in cell metabolism and oxidative stress which regulates 4E-BP), which subsequently activated downstream cell death regulators and resulted in neurodegeneration (such as eye defects and DA neuron loss) in dFoxO overexpressing flies (Kanao et al., 2010). However, it has been found that LRRK2 phosphorylates itself and other substrates more strongly than 4E-BP in mammals (Kumar et al., 2010), so the negative effects may be Drosophila specific. Another study recently reported that dLRRK/hLRRK2 targeted microRNA pathways and that pathogenic LRRK2 antagonized these miRNAs, which negatively affected cell cycle and DA neuron survival (Gehrke et al., 2010). Additionally, the group found that LRRK2 associated with and negatively regulated Argonaute (dAgo1/hAgo2) RISC proteins and LRRK2 mutants promoted the association of phospho-4E-BP1 with hAgo2. Thus, it appears that WT LRRK2 can regulate protein expression by targeting microRNA silencing pathways, and mutations in LRRK2 can upset a delicate balance.

\section{Rodent LRRK2 models}

While invertebrate LRRK2 models have been and will continue to be highly informative, it is important to remember that the LRRK genes are not true orthologues of LRRK2 (Marin, 2008). In addition, physiological limitations include not only the lack of basal ganglia but also their short lifespan, leaving the effects of aging impossible to determine. Given that age is the biggest risk factor for development of LRRK2 parkinsonism, the development of mammalian LRRK2 models is key for understanding pathophysiology and for drug screening.

To date a number of rodent models have been published in the literature, with experimental approaches Including knock-out of LRRK2, over-expression of human wild-type and 
mutant LRRK2, over-expression of mouse wild-type and mutant LRRK2 and targeted knock-in of human mutations into the murine genome.

\subsection{Murine LRRK2 knockouts}

Several groups have reported LRRK2 knockout mice in varying detail. In 2009, Andreas-Mateo et al reported that mice with partial knockout of exon 39 and complete knockout of exon 40 are viable, grossly normal and have completely intact dopaminergic system in terms of dopamine levels and neuronal number up to 2 years of age and show no altered sensitivity to 1-methyl-4phenyl-1,2,3,6-tetrahydropyridine (MPTP) (Andres-Mateos et al., 2009). Around the same time Lin et al also reported that no pathological or behavioral abnormalities were observed in their knockout mice that lack LRRK2 exon 2 (Lin et al., 2010). Interestingly, when they crossed the LRRK2 knockout to conditional A53T mice, a complete amelioration of $\alpha$-synuclein mediated neuropathological abnormalities was observed. The third report which described two lines of knockout mice (one with ablation of exon 1, the other ablation of exons 29/30) also reported a lack of any dopaminergic phenotypes or brain neuropathology (Tong et al., 2010). Surprisingly, a major renal phenotype was found in these mice which included dramatic morphological changes and shrinkage, accumulation of alpha-synuclein and pSer 129 alphasynuclein, impaired ubiquitin degradation, impaired autophagy, apoptosis and inflammation. Interestingly, in a different exon 1 knockout model, yet to be fully described, decreased phospho-tau levels and concurrent increased soluble tubulin levels were reported, implying a role for LRRK2 in regulation microtubule dynamics (Gillardon, 2009).

Our group has also created LRRK2 $\mathrm{KO}$ mice, which are in the final stages of characterization. Microdialysis in these mice has revealed that while the dopamine release following $\mathrm{KCl}$ stimulated is not different in magnitude to the wild-type controls, it appears to be slightly delayed. Starting from about 4 months we have also seen progressive darkening of the kidneys and histopathological analysis reveals severe inflammation.

\subsection{Over-expression of LRRK2}

The first rodent over-expression models were described by McLeod et al in 2006 (MacLeod et al., 2006), whose group investigated both embryonic cDNA electroporation and adult adeno-associated viral expression of WT and mutant LRRK2 kinase domain constructs in rats. A reduction in neurite outgrowth and branching was observed in the embryos expressing mutant G2019S and Y1699C in periventricular layer neural progenitors. In adult rats, viral expression of WT and G2019S in the nigra resulted apotosis in nigral neurons and striatal abnormalities including phospho-tau increases.

Three years later, Chenjian Li's group published the first bacterial artificial chromosome (BAC) mouse models, using human WT and ROC domain mutant R1441G BACs (Li et al., 2009). The mice showed an expression pattern of transgenic LRRK2 that mirrored endogenous expression patterns at levels around 5-fold on an FVB/ N background. Striking phenotypes were reported including progressive levodopa-responsive slowness of movement associated with $\sim 33 \%$ decreased dopamine release following nomifensine treatment and axonal pathology of nigrostriatal dopaminergic projection. While nigral neuronal loss was absent, the R1441G mice displayed a marked reduction in the number of tyrosine hydroxylase-positive nigral dendrites and accumulation of microtubule associated protein tau pathology in the striatum. Extensive characterization of their expression matched human WT BAC model model was not presented, however no overt phenotypes were noted. 
Two groups have since published data on BAC models expressing the kinase domain mutant G2019S. Li et al expressed a murine wild type and mutant G2019S BAC on a C57BL/ 6 background, with expression levels around 6 fold over endogenous LRRK2 (Li et al., 2010). Total dopamine content in the striatum was normal in WT BAC mice and G2019S mice at 6 months but declined by 25\% in G2019S BAC mice by 12 months, suggesting an age related decline. Despite normal striatal dopamine levels, voltammetry in brain slices derived from 12 month old wild-type BAC transgenic mice showed a $25 \%$ increase in evoked dopamine release following a single pulse, whereas the decline in DA release following repeated stimulation was comparable to non-transgenic controls, as was their dopamine transporter (DAT)-mediated uptake. Conversely, the G2019S BAC mice displayed a 35\% decrease in dopamine release by voltammetry, poorly sustained evoked dopamine release after repeated stimulation and a lower rate of dopamine uptake, presumably as a compensatory mechanism. No dopamine neuronal loss was seen in either model. Behaviorally the wild-type transgenic mice displayed enhanced ability on the beam test and increase spontaneous activity, whereas the G2019S BAC mice did not display any abnormalites. While no overt pathological lesions were reported, the authors did reveal that phospho- tau was decreased in the WT transgenic mice.

Our group (Melrose et al., 2010) also reported WT and mutant G2019S BAC mice, utilizing a human LRRK2 BAC rather than a murine BAC. Using in vivo microdialysis we demonstrated that basal extracellular dopamine levels were reduced by $\sim 33 \%$ in G2019S BAC mice at $\sim 8$ months and an enhanced response to amphetamine challenge was noted. Total striatal dopamine, dopamine neuronal number, DAT levels and D1 and D2 receptor density were all unchanged up to 22 months. Behaviorally, the G2019S mice displayed some abnormal open field behavior but no motor deficits. Surprisingly, and in contrast to Li et al, we found that our human WT BAC mice also displayed reduced basal dopamine levels, even more pronounced $(\sim 66 \%$ versus $33 \%)$ than in our G2019S BAC mice. While this is likely attributed to differential expression levels in the two lines (WT BAC mice around 3.5 fold overexpression in half brain extracts and 2.5 fold in G2019S mice) it nevertheless supports a gain of function mechanism. Interestingly, D1 receptors were slightly upregulated in aged human WT BAC mice, although no changes were found in D2 receptors or DAT levels. Pathologically we did not detect any alpha-synuclein pathology in either line, but we did see changes in the phosphorylation of tau. Modest phosphorylation changes, detected by immunohistochemistry, were restricted to the hippocampus in the human WT BAC mice (the area of highest expression). In the G2019S mice however, tau alterations were much more pronounced and widespread. Biochemical analysis of tau revealed enhanced phosphorylation at several phospho-epitopes and upon dephosphorylation the tau species distribution was still different from the non-transgenic controls, suggesting that other post-translational modifications may be altered in G2019S mice and we hypothesize that these processing changes may increase the likelihood of abberant phosphorylation. One final observation worth noting is that we recently showed that neurogenesis is altered in our G2019S mice (Winner et al., 2011). Proliferation and migration of new neurons was impaired in the subventricular zone/rostral migratory stream and in the subgranular zone of the hippocampus. Furthermore, new neurons in the hippocampus had impaired outgrowth and a diminished number of spines. Curiously, exercise was found to partially recover the neuroblast deficit in the hippocampus, which may suggest that exercise induced signaling can at least partially compensate abberant LRRK2 activity caused by the G2019S mutation. 
In a different transgenic approach Lin et al generated hemagglutinin tagged conditional human WT and G2019S using cDNA fragments inserted into the mouse prion protein expression vector controlled by the tetracycline-responsive promoter (tetP) (Lin et al., 2009). Expression levels were between 8-16 fold for the human WT and G2019S lines and mice were on a C57BL/ 6 background. Human WT mice were behaviorally normal, but G2019S mice were found to gain less weight than controls and had increased ambulatory and rearing activities. One month old human WT and G2019S and mice were also reported to have perturbed microtubule dynamics, evidenced by a dramatic shift of $\beta$-tubulin from soluble to insoluble fractions prepared from brain lysates. Finally, ubiquitin staining increased in cortical neurons of 6 month old human WT and G2019S mice and by 20 months this increase became more pronounced and was clustered with LRRK2 staining, which the authors suggest may hint to inhibition of ubiquitin-proteasome activities. Interestingly, both the human WT and G2019S mice enhanced $\alpha$-synuclein pathology when crossed with inducible A53T mutant mice. Curiously, double A53T/ kinase dead G2019S mice also exhibited similar enhanced phenotype, suggesting that kinase activity was not involved in $\alpha$-synuclein mediating toxicity. The authors instead attributed the synergistic effects to impairment of golgi function, microtubule transport and the ubiquitin-proteasomal pathway, which occurred when LRRK2 was co-expressed, regardless of the transgene type or expression level.

Two recently described viral models are the only LRRK2 models to date that document dopamine neuronal loss. Lee et al (Lee et al., 2010a) developed herpes simplex virus (HSV) amplicon-based mouse models expressing either human WT, G2019S and G2019S-D1994A (kinase dead) LRRK2, in which up to 75\% of nigral neurons were infected after injection. HSV amplicon-mediated delivery of LRRK2 G2019S induced significant loss ( 50\%) of tyrosine hydroxylase-positive neurons 3 weeks after stereotaxic injection into the ipsilateral striatum of mice compared to control viruses expressing WT LRRK2 and eGFP and G2019SD1994A. This model is the first model that directly implicates kinase activity being responsible for toxicity in vivo in a mammalian model, supported by the lack of effect in the kinase dead model, as well as attenuation of the neuronal loss by kinase inhibitors GW5074 and indirubin-3'-monooxime. The second viral model, the only published rat model to date, used a second generation adeno-viral serotype 5 vector, with transgene expression driven by the neuronal-specific human synapsin-1 promoter to express human WT and G2019S LRRK2 in rat brain (Dusonchet et al., 2011). Injections were delivered to the striatum and retrograde expression in the nigra was around 2 fold expression level overall, although with only $30 \%$ of all neurons transduced, this suggested very high levels in individual neurons. G2019S, but not WT mice exhibited a progressive neuronal loss that reached $\sim 20 \%$ by 42 days as well as a $10 \%$ decrease in tyrosine hydroxylase fiber density. No alpha- synuclein pathology was observed but the authors noted some transient abnormal phosphorylation of tau for both WT and G2019S which did not correlate with neuronal cell death in G2019S.

\subsection{Targeted knockin of LRRK2}

For large proteins such as LRRK2, the over-expression approach is not without caveat and within the field there has been much concern about over-expession artifacts. To circumvent this, targeting the models' own genome, known as "knockin" is an alternative and potentially more physiological approach, since gene expression is recapitulated in the correct temporal, anatomical and quantitative manner and under the endogenous 
transcriptional and translational controls. Nevertheless, many would still argue, justifiably, that over-expression models and their phenotypes, even if exaggerated, often give vital clues to normal and pathological functions. Equally, the risk with the knockin approach is that the relatively low mutant expression levels, within a species that doesn't normally develop the disease, may yield no or very subtle phenotypes.

To date only one knockin model has been published, by Tong et al, who created a ROC domain R1441C mutant (Tong et al., 2009). Whilst the homozygous knockin mice were grossly normal and did not exhibit dopamine neuronal loss, amperometric recordings in chromaffin cells derived from the $\mathrm{R} 1441 \mathrm{C}$, stimulated with high $\mathrm{KCl}$, revealed significant reductions in the total catecholamine release per cell, quantal size and the frequency of release events. Slice recordings from dopaminergic neurons in the nigra of R1441C mice also exhibited smaller amplitude and duration of hyperpolarization after dopamine application, as well as slower recover times, and reduced responses to amphetamine and the D2 agonist quinpirole, compared with WT controls. Locomotor testing in the open field also revealed the knockin mice had a reduced behavioral response to amphetamine and quinpirole, providing in vivo evidence that dopamine release and D2 autoceptor feedback mechanisms may be impaired. No neuropathology was noted in these mice.

\subsection{What do the models tell us about LRRK2?}

At first glance, the success of the LRRK2 models appears to be nominal, with not a single model recapturing all the key features desired for a PD model - dopamine cell loss, motor impairment and alpha-synculein pathology. However, independent of the approach, a unifying theme is emerging from both invertebrate and vertebrate LRRK2 models, suggesting an important role for LRRK2 in dopaminergic neurotransmission, even in the absence of dopamine neuronal loss. Several other genetic PD mouse models also have abnormalities in dopamine transmission without neuronal loss including PINK1, parkin, DJ1 and SNCA knockouts and SNCA WT over-expression mice (Abeliovich et al., 2000; Goldberg et al., 2003; Goldberg et al., 2005; Kitada et al., 2009; Nemani et al., 2010). Imaging studies have long established that in asymptomatic PD, the earliest detectable changes occur in the dopamine transporter and the same holds true for asymptomatic LRRK2 (Nandhagopal et al., 2008; Sossi et al., 2010) and SNCA patients (Bostantjopoulou et al., 2008; Perani et al., 2006; Samii et al., 1999). Thus, the data evolving from LRRK2 models (and other PD genetic models) may be recapturing early preclinical events. In reality this may make the models more valuable because the onset of motor symptoms in PD is only after 50$70 \%$ of the dopamine neurons are lost, by which point neuro-protective therapy would be futile. Understanding these early events in disease is key to allow us identify biomarkers to detect at risk patients and design neuro-protective therapies.

Aside from the effects on dopamine neurotransmission, the other consistent theme arising from LRRK2 in vivo data appears to be the impairment of cytoskeletal function, evidenced by the alterations observed in tau phosphorylation and perturbed microtubule dynamics. Although tau pathology is rare in LRRK2 brains, there is mounting evidence of the involvement of tau in PD in general, with over 15 genetic studies in small populations implicating variability in the tau gene with Parkinson's disease and a recent study of a large number of European PD samples unequivocally showing that tau gene (MAPT) variability is a major risk factor for PD, along with the alpha-synculein gene (SNCA) (Simon-Sanchez et al., 2009). Even though it has been proven already that there is no direct interaction between 
tau and LRRK2 protein, there appears to be an indirect link that warrants further investigation. If LRRK2 regulates tau physiology, this could have important implication for LRRK2 therapies in Alzheimer's disease.

Less consistent/investigated in vivo, but nevertheless still compelling are the changes in the inflammatory, autophagy/lysosomal, apoptotic, mitochondrial and proteosomal pathways observed in some models. These pathways may be particularly important in unraveling how LRRK2 leads to $\alpha$-synuclein pathology in humans. While studies in invertebrates are suggestive of a potential role for LRRK2, more mammalian studies are needed into the role of LRRK2 in mitochrondrial/stress pathways. As it stands, there is no evidence for a direct interaction between LRRK2 and $\alpha$-synuclein and the only instance of in vivo $\alpha$-synuclein pathology in a LRRK2 model is in the kidneys, but not brain, of the LRRK2 knockout model (Tong et al., 2010). Notably, accumulation was accompanied by autophagic, proteasomal and inflammatory changes. Furthermore in double LRRK2 and $\alpha$-synuclein A53T mice, enhancement of $\alpha$-synuclein pathology was attributed to impairments in microtubule dynamic, golgi organization, mitochondrial toxicity and ubiquitin-proteasomal pathway (Lin et al., 2009). What is difficult to resolve though, is that double LRRK2 KO/ A53T mice have ameliorated $\alpha$-synuclein brain pathology (Lin et al., 2009). These complex findings reiterate the notion that LRRK2 is likely a multi-faceted protein, which may have distinct cellular specific roles.

\subsection{The future for LRRK2 therapeutics}

Intense pharmaceutical interest has surrounded LRRK2 since its discovery and many companies are developing inhibitors of LRRK2 and screening is already underway in many of the models described above. It is still not clear exactly what function of LRRK2 mediates its toxicity and data attributing it to enzymatic kinase activity is conflicting. Although the general consensus appears to be that LRRK2 pathogenicity is a toxic gain of function, the kidney phenotype in LRRK2 KO mice suggests that peripheral effects may be an important loss-of-function consideration. A highly selective LRRK2 inhibitor (LRRK2-IN1) was recently developed by Nathaniel Gray and colleagues (Deng et al., 2011) which abolished Ser910 and Ser935 phosphorylation of LRRK2 in the kidney of mice after 1 hour. No changes were observed in brain because the compound is unable to cross the blood brain barrier. Once CNS drug delivery issues are overcome, it will be interesting to see the effects of LRRK-IN1in in vivo LRRK2 models.

\section{References}

Aasly, J. O., et al., 2005. Clinical features of LRRK2-associated Parkinson's disease in central Norway. Ann Neurol. 57, 762-5.

Abeliovich, A., et al., 2000. Mice lacking alpha-synuclein display functional deficits in the nigrostriatal dopamine system. Neuron. 25, 239-52.

Adams, J. R., et al., 2005. PET in LRRK2 mutations: comparison to sporadic Parkinson's disease and evidence for presymptomatic compensation. Brain. 128, 2777-85.

Andres-Mateos, E., et al., 2009. Unexpected lack of hypersensitivity in LRRK2 knock-out mice to MPTP (1-methyl-4-phenyl-1,2,3,6-tetrahydropyridine). J Neurosci. 29, 15846-50. 
Biskup, S., et al., 2006. Localization of LRRK2 to membranous and vesicular structures in mammalian brain. Ann Neurol. 60, 557-69.

Biskup, S., et al., 2007. Dynamic and redundant regulation of LRRK2 and LRRK1 expression. BMC Neurosci. 8, 102.

Bostantjopoulou, S., et al., 2008. (123)I-FP-CIT SPET striatal uptake in parkinsonian patients with the alpha-synuclein (G209A) mutation A. Hell J Nucl Med. 11, 157-9.

Brand, A. H., Perrimon, N., 1993. Targeted gene expression as a means of altering cell fates and generating dominant phenotypes. Development. 118, 401-15.

Carmine Belin, A., et al., 2006. Leucine-rich repeat kinase 2 (LRRK2) mutations in a Swedish Parkinson cohort and a healthy nonagenarian. Mov Disord. 21, 1731-4.

Dachsel, J. C., et al., 2007. Lrrk2 G2019S substitution in frontotemporal lobar degeneration with ubiquitin-immunoreactive neuronal inclusions. Acta Neuropathol. 113, 6016.

Deng, X., et al., 2011. Characterization of a selective inhibitor of the Parkinson's disease kinase LRRK2. Nat Chem Biol. 7, 203-5.

Di Fonzo, A., et al., 2006. Comprehensive analysis of the LRRK2 gene in sixty families with Parkinson's disease. Eur J Hum Genet. 14, 322-31.

Djaldetti, R., et al., 2008. Clinical characteristics of Parkinson's disease among Jewish Ethnic groups in Israel. J Neural Transm. 115, 1279-84.

Dusonchet, J., et al., 2011. A rat model of progressive nigral neurodegeneration induced by the Parkinson's disease-associated G2019S mutation in LRRK2. J Neurosci. 31, 90712.

Farrer, M. J., et al., 2007. Lrrk2 G2385R is an ancestral risk factor for Parkinson's disease in Asia. Parkinsonism Relat Disord. 13, 89-92.

Galter, D., et al., 2006. LRRK2 expression linked to dopamine-innervated areas. Ann Neurol. $59,714-9$.

Gehrke, S., et al., 2010. Pathogenic LRRK2 negatively regulates microRNA-mediated translational repression. Nature. 466, 637-41.

Gillardon, F., 2009. Leucine-rich repeat kinase 2 phosphorylates brain tubulin-beta isoforms and modulates microtubule stability--a point of convergence in parkinsonian neurodegeneration? J Neurochem. 110, 1514-22.

Goldberg, M. S., et al., 2003. Parkin-deficient mice exhibit nigrostriatal deficits but not loss of dopaminergic neurons. J Biol Chem. 278, 43628-35.

Goldberg, M. S., et al., 2005. Nigrostriatal dopaminergic deficits and hypokinesia caused by inactivation of the familial Parkinsonism-linked gene DJ-1. Neuron. 45, 48996.

Greggio, E., et al., 2006. Kinase activity is required for the toxic effects of mutant LRRK2/dardarin. Neurobiol Dis. 23, 329-41.

Greggio, E., et al., 2008. The Parkinson disease-associated leucine-rich repeat kinase 2 (LRRK2) is a dimer that undergoes intramolecular autophosphorylation. J Biol Chem. 283, 16906-14.

Healy, D. G., et al., 2008. Phenotype, genotype, and worldwide genetic penetrance of LRRK2-associated Parkinson's disease: a case-control study. Lancet Neurol. 7, 58390. 
Higashi, S., et al., 2007a. Localization of Parkinson's disease-associated LRRK2 in normal and pathological human brain. Brain Res. 1155, 208-19.

Higashi, S., et al., 2007b. Expression and localization of Parkinson's disease-associated leucine-rich repeat kinase 2 in the mouse brain. J Neurochem. 100, 368-81.

Hulihan, M. M., et al., 2008. LRRK2 Gly2019Ser penetrance in Arab-Berber patients from Tunisia: a case-control genetic study. Lancet Neurol. 7, 591-4.

Imai, Y., et al., 2008. Phosphorylation of 4E-BP by LRRK2 affects the maintenance of dopaminergic neurons in Drosophila. EMBO J. 27, 2432-43.

Ishihara, L., et al., 2007. Screening for Lrrk2 G2019S and clinical comparison of Tunisian and North American Caucasian Parkinson's disease families. Mov Disord. 22, 55-61.

Jaleel, M., et al., 2007. LRRK2 phosphorylates moesin at threonine-558: characterization of how Parkinson's disease mutants affect kinase activity. Biochem J. 405, 30717.

Kachergus, J., et al., 2005. Identification of a novel LRRK2 mutation linked to autosomal dominant parkinsonism: evidence of a common founder across European populations. Am J Hum Genet. 76, 672-80.

Kanao, T., et al., 2010. Activation of FoxO by LRRK2 induces expression of proapoptotic proteins and alters survival of postmitotic dopaminergic neuron in Drosophila. Hum Mol Genet. 19, 3747-58.

Kay, D. M., et al., 2005. Escaping Parkinson's disease: a neurologically healthy octogenarian with the LRRK2 G2019S mutation. Mov Disord. 20, 1077-8.

Kitada, T., et al., 2009. Impaired dopamine release and synaptic plasticity in the striatum of parkin-/- mice. J Neurochem. 110, 613-21.

Kumar, A., et al., 2010. The Parkinson's disease associated LRRK2 exhibits weaker in vitro phosphorylation of 4E-BP compared to autophosphorylation. PLoS One. 5, e8730.

Lee, B. D., et al., 2010a. Inhibitors of leucine-rich repeat kinase-2 protect against models of Parkinson's disease. Nat Med. 16, 998-1000.

Lee, S., et al., 2010b. LRRK2 kinase regulates synaptic morphology through distinct substrates at the presynaptic and postsynaptic compartments of the Drosophila neuromuscular junction. J Neurosci. 30, 16959-69.

Lee, S. B., et al., 2007. Loss of LRRK2/PARK8 induces degeneration of dopaminergic neurons in Drosophila. Biochem Biophys Res Commun. 358, 534-9.

Li, X., et al., 2007. Leucine-rich repeat kinase 2 (LRRK2)/PARK8 possesses GTPase activity that is altered in familial Parkinson's disease R1441C/G mutants. J Neurochem. $103,238-47$.

Li, Y., et al., 2009. Mutant LRRK2(R1441G) BAC transgenic mice recapitulate cardinal features of Parkinson's disease. Nat Neurosci. 12, 826-8.

Lin, C. H., et al., 2010. LRRK2 G2019S mutation induces dendrite degeneration through mislocalization and phosphorylation of tau by recruiting autoactivated GSK3ss. J Neurosci. 30, 13138-49. 
Lin, X., et al., 2009. Leucine-rich repeat kinase 2 regulates the progression of neuropathology induced by Parkinson's-disease-related mutant alpha-synuclein. Neuron. 64, 80727.

Liu, Z., et al., 2008. A Drosophila model for LRRK2-linked parkinsonism. Proc Natl Acad Sci U S A. 105, 2693-8.

Luzon-Toro, B., et al., 2007. Mechanistic insight into the dominant mode of the Parkinson's disease-associated G2019S LRRK2 mutation. Hum Mol Genet. 16, 2031-9.

MacLeod, D., et al., 2006. The familial Parkinsonism gene LRRK2 regulates neurite process morphology. Neuron. 52, 587-93.

Marin, I., 2008. Ancient origin of the Parkinson disease gene LRRK2. J Mol Evol. 67, 4150.

Mata, I. F., et al., 2005. Lrrk2 pathogenic substitutions in Parkinson's disease. Neurogenetics. $6,171-7$.

Mata, I. F., et al., 2006. LRRK2 mutations are a common cause of Parkinson's disease in Spain. Eur J Neurol. 13, 391-4.

Melrose, H., et al., 2006. Anatomical localization of leucine-rich repeat kinase 2 in mouse brain. Neuroscience. 139, 791-4.

Melrose, H. L., et al., 2010. Impaired dopaminergic neurotransmission and microtubuleassociated protein tau alterations in human LRRK2 transgenic mice. Neurobiol Dis. 40, 503-17.

Melrose, H. L., et al., 2007. A comparative analysis of leucine-rich repeat kinase 2 (Lrrk2) expression in mouse brain and Lewy body disease. Neuroscience. 147, 1047-58.

Miklossy, J., et al., 2006. LRRK2 expression in normal and pathologic human brain and in human cell lines. J Neuropathol Exp Neurol. 65, 953-63.

Nandhagopal, R., et al., 2008. Progression of dopaminergic dysfunction in a LRRK2 kindred: a multitracer PET study. Neurology. 71, 1790-5.

Nemani, V. M., et al., 2010. Increased expression of alpha-synuclein reduces neurotransmitter release by inhibiting synaptic vesicle reclustering after endocytosis. Neuron. 65, 66-79.

$\mathrm{Ng}$, C. H., et al., 2009. Parkin protects against LRRK2 G2019S mutant-induced dopaminergic neurodegeneration in Drosophila. J Neurosci. 29, 11257-62.

Ozelius, L. J., et al., 2006. LRRK2 G2019S as a cause of Parkinson's disease in Ashkenazi Jews. N Engl J Med. 354, 424-5.

Paisan-Ruiz, C., et al., 2004. Cloning of the gene containing mutations that cause PARK8linked Parkinson's disease. Neuron. 44, 595-600.

Perani, D., et al., 2006. Positron emission tomography changes in PARK1 mutation. Mov Disord. 21, 127-30.

Rajput, A., et al., 2006. Parkinsonism, Lrrk2 G2019S, and tau neuropathology. Neurology. 67, 1506-8.

Ross, O. A., et al., 2006. Lrrk2 and Lewy body disease. Ann Neurol. 59, 388-93.

Ross, O. A., et al., 2008. Analysis of Lrrk2 R1628P as a risk factor for Parkinson's disease. Ann Neurol. 64, 88-92. 
Saha, S., et al., 2009. LRRK2 modulates vulnerability to mitochondrial dysfunction in Caenorhabditis elegans. J Neurosci. 29, 9210-8.

Sakaguchi-Nakashima, A., et al., 2007. LRK-1, a C. elegans PARK8-related kinase, regulates axonal-dendritic polarity of SV proteins. Curr Biol. 17, 592-8.

Samann, J., et al., 2009. Caenorhabditits elegans LRK-1 and PINK-1 act antagonistically in stress response and neurite outgrowth. J Biol Chem. 284, 16482-91.

Samii, A., et al., 1999. PET studies of parkinsonism associated with mutation in the alphasynuclein gene. Neurology. 53, 2097-102.

Simon-Sanchez, J., et al., 2006. LRRK2 is expressed in areas affected by Parkinson's disease in the adult mouse brain. Eur J Neurosci. 23, 659-66.

Simon-Sanchez, J., et al., 2009. Genome-wide association study reveals genetic risk underlying Parkinson's disease. Nat Genet. 41, 1308-12.

Smith, W. W., et al., 2006. Kinase activity of mutant LRRK2 mediates neuronal toxicity. Nat Neurosci. 9, 1231-3.

Sossi, V., et al., 2010. Dopamine turnover increases in asymptomatic LRRK2 mutations carriers. Mov Disord. 25, 2717-23.

Tain, L. S., et al., 2009. Rapamycin activation of 4E-BP prevents parkinsonian dopaminergic neuron loss. Nat Neurosci. 12, 1129-35.

Tan, E. K., 2006. Identification of a common genetic risk variant (LRRK2 Gly2385Arg) in Parkinson's disease. Ann Acad Med Singapore. 35, 840-2.

Taylor, J. P., et al., 2006. LRRK2: a common pathway for parkinsonism, pathogenesis and prevention? Trends Mol Med. 12, 76-82.

Taymans, J. M., et al., 2006. Distribution of PINK1 and LRRK2 in rat and mouse brain. J Neurochem. 98, 951-61.

Tong, Y., et al., 2009. R1441C mutation in LRRK2 impairs dopaminergic neurotransmission in mice. Proc Natl Acad Sci U S A. 106, 14622-7.

Tong, Y., et al., 2010. Loss of leucine-rich repeat kinase 2 causes impairment of protein degradation pathways, accumulation of alpha-synuclein, and apoptotic cell death in aged mice. Proc Natl Acad Sci U S A. 107, 9879-84.

Venderova, K., et al., 2009. Leucine-Rich Repeat Kinase 2 interacts with Parkin, DJ-1 and PINK-1 in a Drosophila melanogaster model of Parkinson's disease. Hum Mol Genet. 18, 4390-404.

Wang, D., et al., 2008. Dispensable role of Drosophila ortholog of LRRK2 kinase activity in survival of dopaminergic neurons. Mol Neurodegener. 3, 3.

West, A. B., et al., 2005. Parkinson's disease-associated mutations in leucine-rich repeat kinase 2 augment kinase activity. Proc Natl Acad Sci U S A. 102, 16842-7.

Westerlund, M., et al., 2008. Developmental regulation of leucine-rich repeat kinase 1 and 2 expression in the brain and other rodent and human organs: Implications for Parkinson's disease. Neuroscience. 152, 429-36.

White, L. R., et al., 2007. MAPK-pathway activity, Lrrk2 G2019S, and Parkinson's disease. J Neurosci Res. 85, 1288-94.

Winner, B., et al., 2011. Adult neurogenesis and neurite outgrowth are impaired in LRRK2 G2019S mice. Neurobiol Dis. 41, 706-16. 
Wolozin, B., et al., 2008. Investigating convergent actions of genes linked to familial Parkinson's disease. Neurodegener Dis. 5, 182-5.

Wszolek, ZK et al., 2004. Autosomal dominant parkinsonism associated with variable synuclein and tau pathology. Neurology. 62(9):1619-22.

Yao, C., et al., 2010. LRRK2-mediated neurodegeneration and dysfunction of dopaminergic neurons in a Caenorhabditis elegans model of Parkinson's disease. Neurobiol Dis. 40, 73-81.

Zimprich, A., et al., 2004. Mutations in LRRK2 cause autosomal-dominant parkinsonism with pleomorphic pathology. Neuron. 44, 601-7. 




\author{
Etiology and Pathophysiology of Parkinson's Disease \\ Edited by Prof. Abdul Qayyum Rana
}

ISBN 978-953-307-462-7

Hard cover, 542 pages

Publisher InTech

Published online 12, October, 2011

Published in print edition October, 2011

This book about Parkinsonâ $€^{\mathrm{TM}} \mathrm{S}$ disease provides a detailed account of etiology and pathophysiology of Parkinsonâ€ ${ }^{\mathrm{TM}} \mathrm{s}$ disease, a complicated neurological condition. Environmental and genetic factors involved in the causation of Parkinsonâ€ ${ }^{\mathrm{TM}} \mathrm{S}$ disease have been discussed in detail. This book can be used by basic scientists as well as researchers. Neuroscience fellows and life science readers can also obtain sufficient information. Beside genetic factors, other pathophysiological aspects of Parkinsonâ $€^{\mathrm{TM}} \mathrm{s}$ disease have been discussed in detail. Up to date information about the changes in various neurotransmitters, inflammatory responses, oxidative pathways and biomarkers has been described at length. Each section has been written by one or more faculty members of well known academic institutions. Thus, this book brings forth both clinical and basic science aspects of Parkinsonâ ${ }^{\mathrm{TM}} \mathrm{S}$ disease.

\title{
How to reference
}

In order to correctly reference this scholarly work, feel free to copy and paste the following:

Kelly Hinkle and Heather Melrose (2011). Modeling LRRK2 Parkinsonism, Etiology and Pathophysiology of Parkinson's Disease, Prof. Abdul Qayyum Rana (Ed.), ISBN: 978-953-307-462-7, InTech, Available from: http://www.intechopen.com/books/etiology-and-pathophysiology-of-parkinson-s-disease/modeling-Irrk2parkinsonism

\section{INTECH}

open science | open minds

\author{
InTech Europe \\ University Campus STeP Ri \\ Slavka Krautzeka 83/A \\ 51000 Rijeka, Croatia \\ Phone: +385 (51) 770447 \\ Fax: +385 (51) 686166 \\ www.intechopen.com
}

\author{
InTech China \\ Unit 405, Office Block, Hotel Equatorial Shanghai \\ No.65, Yan An Road (West), Shanghai, 200040, China \\ 中国上海市延安西路65号上海国际贵都大饭店办公楼 405 单元 \\ Phone: +86-21-62489820 \\ Fax: $+86-21-62489821$
}


(C) 2011 The Author(s). Licensee IntechOpen. This is an open access article distributed under the terms of the Creative Commons Attribution 3.0 License, which permits unrestricted use, distribution, and reproduction in any medium, provided the original work is properly cited. 